\title{
World Journal of
Pediatric Surgery Survey on antibiotic regimens for necrotizing enterocolitis prescribed by Chinese pediatricians in 2020
}

Zhongyao Zhang, Yu He, Zhengli Wang, Lei Bao, Yuan Shi, Luquan Li

To cite: Zhang Z, He Y, Wang Z, et al. Survey on antibiotic regimens for necrotizing enterocolitis prescribed by Chinese pediatricians in 2020. World Jnl Ped Surgery 2021;4:e000253. doi:10.1136/ wjps-2020-000253

- Additional supplemental material is published online only. To view, please visit the journal online (http://dx.doi.org/10.1136/ wjps-2020-000253).

Received 24 December 2020 Revised 8 April 2021 Accepted 15 April 2021
Check for updates

(C) Author(s) (or their employer(s)) 2021. Re-use permitted under CC BY-NC. No commercial re-use. See rights and permissions. Published by BMJ.

Neonatal Diagnosis and Treatment Center, Chongqing Medical University Affiliated Children's Hospital, Chongqing, China

Correspondence to Dr Luquan Li; liluquan123@ 163.com

\section{ABSTRACT}

Background Necrotizing enterocolitis (NEC) is a serious intestinal inflammatory disease in neonates, and intravenous antibiotics constitute the main therapeutic strategy. Studies have shown that substantial variation in the selection of antibiotic regimens for NEC remains in many countries. The variability in antibiotic therapy selection in China is unclear.

Methods A cross-sectional study using an online questionnaire regarding antibiotic regimens for NEC was conducted among pediatricians working in tertiary hospitals in China.

Results A total of 284 pediatricians from 29 provinces completed the survey; $37.9 \%$ of them administered one antibiotic, $56.7 \%$ administered two antibiotics and $2.4 \%$ administered three antibiotics. The top three single-antibiotic regimens for NEC were beta-lactamase inhibitors $(n=66,41.5 \%)$, carbapenems $(n=46,28.9 \%)$ and cephalosporins ( $n=33,20.8 \%)$. Twenty combinations of two antibiotics were identified, and the top three combinations were beta-lactamase inhibitors and antianaerobic agents $(n=49,19.6 \%)$, carbapenems and glycopeptides $(n=42$, $16.8 \%$ ), and cephalosporins and antianaerobic agents $(n=37,14.8 \%)$. Regarding the therapeutic duration of antibiotic treatment, $77.5 \%(n=220)$ of the pediatricians chose $5-10$ days for stage II NEC, and $79.6 \%(n=226)$ chose 7-14 days for stage III NEC. Forty-three percent $(n=122)$ of the respondents preferred to use carbapenems when NEC was diagnosed, and $83.3 \%(n=135)$ adjusted the antibiotics to carbapenems if a patient's medical condition deteriorated or if the therapeutic efficacy was poor.

Conclusion This survey revealed that carbapenems were the most common antibiotics selected when NEC was diagnosed or a patient's condition deteriorated, but the duration of the course of treatment for NEC varied substantially among doctors.

\section{INTRODUCTION}

Necrotizing enterocolitis (NEC) is an acquired neonatal intestinal disease characterized by severe inflammation attributed to a variety of factors. ${ }^{1}$ NEC is a major cause of morbidity and mortality in the neonatal period. The incidence rate of NEC is $7 \%-13 \%$ in preterm infants younger than 28 weeks of gestational age. ${ }^{2}$ Overall mortality rates due to NEC range from $15 \%$ to $30 \%{ }^{3}$
Summary box

What is already known about this subject

$>$ Necrotizing enterocolitis (NEC) is a serious intestinal inflammatory disease in neonates.

- Intravenous antibiotics constitute the main therapeutic strategy.

- The selection of antibiotic regimens for NEC varies in many countries, and the preferences of Chinese pediatricians are not clear.

What are the new findings

- Carbapenems were the most common antibiotics selected if NEC was diagnosed or if a patient's condition deteriorated.

- The duration of the course of treatment for NEC varied substantially among doctors.

How might it impact on clinical practice in the foreseeable future

- There is no unified antibiotic treatment regimen for $\mathrm{NEC}$, and it is necessary to carry out multicenter research to provide the basis for the formulation of relevant guidelines for the antibiotic treatment of NEC in the future.

Survivors can have significant sequelae, including short-term outcomes, such as intestinal stenosis, short gut syndrome and intestinal failure, ${ }^{4}$ and long-term sequelae, such as growth retardation and severe behavioral and neurodevelopmental delays. ${ }^{5}{ }^{6}$ The current treatment strategy for NEC is a combination of medical and surgical therapies. Non-operative management typically includes bowel rest, gastrointestinal decompression, broad-spectrum intravenous antibiotics, and intravenous nutrition. ${ }^{7}$ Antibiotic treatment is the most important therapeutic strategy. The selection of antibiotics differs in different regions despite the issuance of the American Surgical Infection Society Guidelines in $2010 .{ }^{8}$ In addition, a Cochrane systematic review indicated that there remains a lack of adequate evidence to recommend a particular antibiotic regimen for $\mathrm{NEC},{ }^{9}$ and there is still high variability among the 
antibiotic therapies administered. ${ }^{9-11}$ Blackwood's singlecenter survey showed that up to 22 different antibiotics were used before surgery for NEC. ${ }^{11}$ An international survey conducted in 29 countries indicated that the majority $(67 \%)$ of surgeons chose to treat NEC with a combination of two or more antibiotics. ${ }^{10}$ A retrospective study found that infants with NEC treated with medical (vs surgical) therapy were more likely to be treated with metronidazole, whereas those treated with surgical (vs medical) therapy were more likely to receive vancomycin. ${ }^{12}$ Selected antibiotic regimens vary widely among different specialties and geographic locations ${ }^{10-13}$; however, reports from China are rare, the preferences of Chinese pediatricians in the selection of antibiotics are not clear. Furthermore, there is no clear guideline outlining an optimal therapy strategy for NEC.

The aim of the present study was to investigate the current status of antibiotic regimen selection for the treatment of NEC in China via an online questionnaire. The goals were to obtain expert consensus, to standardize treatment and to update therapeutic recommendations.

\section{METHODS}

\section{Participants}

The study objects included pediatricians in tertiary hospitals in China, who were attending physicians or had more senior positions.

\section{Questionnaire design}

A cross-sectional study was conducted to design the NEC management questionnaire. The questionnaire was divided into three parts: baseline information, NEC diagnosis and NEC treatment. The diagnosis of NEC was based on Bell staging. ${ }^{14}$ The stage II NEC criteria include clinical manifestations, such as abdominal distension; vomiting or blood in the stool (with an absence of fissure); and pneumatosis intestinalis or portal vein gas on X-ray or ultrasound examination. Stage III NEC is diagnosed according to the above criteria plus imaging findings of pneumoperitoneum or a large amount of ascites or the need for surgery when medical therapy is not effective within 48 hours. We sent the questionnaire via a web-based tool (www.wjx.cn) to participants. The questionnaire was completed online on mobile phones or computers, and each computer or mobile phone could only be used to complete the questionnaire once.

\section{Data collection}

Data were collected by an online questionnaire platform (www.wjx.cn). A large-scale network academic conference on neonatal NEC was organized. The participating physicians from tertiary hospitals across the country voluntarily completed the questionnaire through the published links. Thirty-nine participants were excluded because the antibiotic treatment practices were not clear (the type of antibiotic used was not specified; thus, it

\begin{tabular}{|c|c|c|}
\hline Contents & $\mathbf{n}$ & Frequency $(\%)$ \\
\hline \multicolumn{3}{|l|}{ Educational attainment } \\
\hline Undergraduate & 1 & 0.4 \\
\hline Bachelor's degree & 125 & 44.0 \\
\hline Master's degree & 109 & 38.4 \\
\hline Doctor's degree & 49 & 17.3 \\
\hline \multicolumn{3}{|l|}{ Title } \\
\hline Attending physician & 81 & 28.5 \\
\hline Associate chief physician & 83 & 29.2 \\
\hline Chief physician & 120 & 42.3 \\
\hline \multicolumn{3}{|c|}{ Duration of clinical experience $(y)$} \\
\hline$<10$ & 48 & 16.9 \\
\hline $10-20$ & 85 & 29.9 \\
\hline$>20$ & 151 & 53.2 \\
\hline \multicolumn{3}{|c|}{ Number of NEC cases received per year in your hospital } \\
\hline$<50$ & 222 & 78.2 \\
\hline $50-100$ & 39 & 13.7 \\
\hline $100-200$ & 13 & 4.6 \\
\hline$>200$ & 10 & 3.5 \\
\hline \multicolumn{3}{|c|}{ Can NEC surgery be performed in your hospital? } \\
\hline Yes & 109 & 38.4 \\
\hline No & 175 & 61.6 \\
\hline
\end{tabular}

NEC, necrotizing enterocolitis.

could not be classified). We attempted to contact them all by email, but no one replied.

\section{Statistical analysis}

All analyses were performed with SPSS (V.17; SPSS). Categorical variables are expressed as number and percentages $(\%)$. The $\chi^{2}$ test was used for comparisons between groups, and $\mathrm{p}<0.05$ was considered statistically significant. Data were plotted with GraphPad Prism (V.8.0).

\section{RESULTS}

\section{Baseline information analyses}

The survey was administered from March to April 2020. A total of 323 questionnaires were returned, of which 39 were excluded because the use of antibiotics was not clear (the type of antibiotic used was not specified; thus, it could not be classified). A total of 284 questionnaires were finally analyzed (table 1). Participants included 284 pediatricians from 182 tertiary medical units, 89 cities, and 29 provinces in China. Among the surveyed medical units, $96.7 \%$ were equipped with neonatal intensive care units (NICUs), $84.1 \%$ had a separate Department of Neonatology, and $56.0 \%$ had the capacity to perform neonatal surgery and were qualified to perform surgery for NEC. Among the 284 respondents, 28.5\% were attending physicians, $29.2 \%$ were associate chief physicians, and $42.3 \%$ were chief physicians; $55.6 \%$ of the 


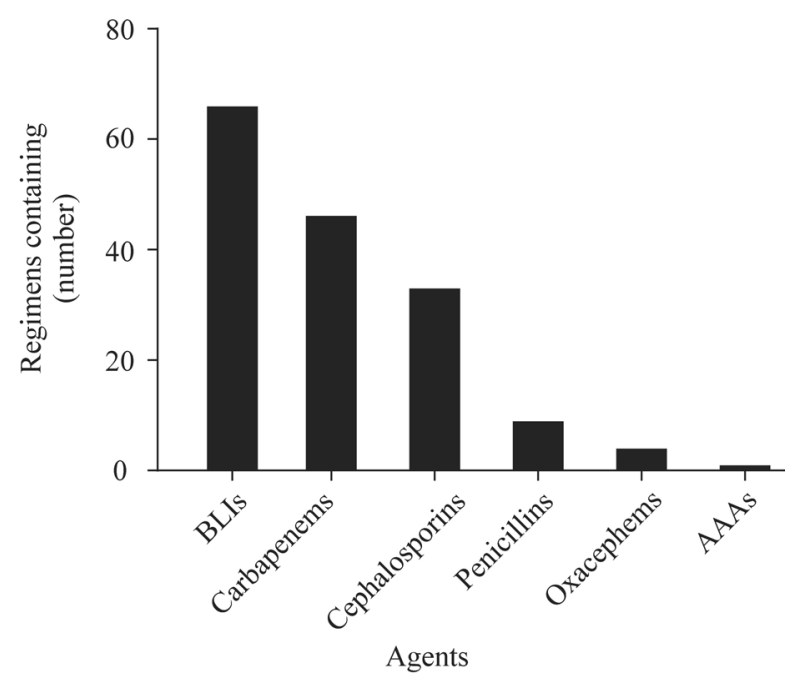

Figure 1 Frequency of single agents. AAAs, antianaerobic agents; BLIs, beta-lactamase inhibitors.

respondents had a master's degree or above. A total of $236(83.1 \%)$ pediatricians had more than 10 years of clinical work experience, and 151 (53.2\%) pediatricians had more than 20 years of experience.

\section{Antibiotic strategy selection}

When NEC was confirmed, 37.9\% of the physicians selected one antibiotic, $56.7 \%$ selected a combination of two antibiotics, and $2.4 \%$ chose three antibiotics. Sixty-six $(41.5 \%)$ physicians chose beta-lactamase inhibitors, $46(28.9 \%)$ chose carbapenems, and 33 (20.8\%) chose cephalosporins, followed by penicillins, oxacephems, and antianaerobic agents. The selection of antibiotics is shown in figure 1 .

Figure 1 showed that beta-lactamase inhibitors and the two other antibiotics had relatively higher selection frequencies. Therefore, this study compared the selection of the top three most commonly selected antibiotics by pediatricians with different durations of clinical experience. The results indicated that there was no significant difference in the selection preferences among doctors with different durations of clinical experience (table 2).

A total of 20 combinations were reported. Forty-nine $(19.6 \%)$ were combinations of beta-lactamase inhibitors and antianaerobic agents, $42(16.8 \%)$ were combinations of carbapenems and glycopeptides, 37 (14.8\%) were combinations of cephalosporins and antianaerobic agents, and $28(11.2 \%)$ were combinations of cephalosporins and penicillins. The top nine most common antibiotic combinations are shown in figure 2. The 20



Figure 2 Frequency of two-antibiotic combinations. AAAs, antianaerobic agents; BLIs, beta-lactamase inhibitors; GLPs, glycopeptides; PENs, penicillins.

combinations of antibiotics are shown in online supplemental table 1.

Figure 2 showed that the selection frequencies of the top five antibiotics, such as beta-lactamase inhibitors and antianaerobic agents, were relatively high. Further comparison of the selections made by physicians with different durations of clinical experience showed no significant differences in the preferences for antibiotic combinations between the groups (table 3 ).

Some participants chose triple antibiotic therapy to treat NEC, including five reports of penicillins, cephalosporins, and antianaerobic agents; three reports of carbapenems, glycopeptides, and antianaerobic agents; and one report of beta-lactamase inhibitors/glycopeptides, antianaerobic agents, and cephalosporins.

\section{Antibiotic duration selection}

The durations of antibiotic therapy selected by the 284 participants for the treatment of stage II NEC are shown in figure 3 . As shown in figure $3,52.1 \%$ of the respondents chose 7-10 days, and similar proportions of the respondents chose 5-7 and 10-14 days. There was no significant difference in the duration of antibiotic courses selected by physicians with different seniority levels (online supplemental table 2).

The durations of antibiotic therapy selected for stage III NEC are shown in figure $3 ; 56.3 \%$ of the respondents chose $7-10$ days, and $23.2 \%$ of the physicians chose 10-14 days. There was no significant difference among

Table 2 Comparison of single agents selected by pediatricians with different seniority levels

\begin{tabular}{lccccc}
\hline Medications, $\mathbf{n}(\%)$ & $<\mathbf{1 0} \mathbf{y}(\mathbf{n}=\mathbf{2 8})$ & $\mathbf{1 0 - 2 0} \mathbf{y}(\mathbf{n = 5 1 )}$ & $\mathbf{> 2 0} \mathbf{y}(\mathbf{n}=\mathbf{6 6})$ & $\boldsymbol{\chi}^{\mathbf{2}}$ & $\mathbf{P}$ value \\
\hline Beta-lactamase inhibitors & $16(57.1)$ & $18(35.3)$ & $32(48.5)$ & 4.345 & 0.361 \\
Carbapenems & $6(21.4)$ & $19(37.3)$ & $21(31.8)$ & & \\
Cephalosporins & $6(21.4)$ & $14(27.5)$ & $13(19.7)$ & & \\
\hline
\end{tabular}


Table 3 Comparison of the combinations of two antibiotics selected by physicians with different seniority levels

\begin{tabular}{|c|c|c|c|c|c|}
\hline Medications, n (\%) & $<10$ y $(n=25)$ & $10-20$ y $(n=50)$ & $>20$ y $(n=105)$ & $\chi^{2}$ & $P$ value \\
\hline BLIs+AAAs & $9(36.0)$ & $16(32.0)$ & 24 (22.9) & 8.246 & 0.410 \\
\hline Carbapenems+GLPs & $8(32.0)$ & $9(18.0)$ & $25(23.8)$ & & \\
\hline Cephalosporins+AAAs & $5(20.0)$ & $11(22.0)$ & $21(20.0)$ & & \\
\hline Cephalosporins+PENs & $0(0)$ & $8(16.0)$ & 20 (19.0) & & \\
\hline Carbapenems+AAAs & $3(12.0)$ & $6(12.0)$ & 15 (14.3) & & \\
\hline
\end{tabular}

AAAs, antianaerobic agents; BLIs, beta-lactamase inhibitors; GLPs, glycopeptides; PENs, penicillins.

physicians with different seniority levels (online supplemental table 3).

\section{Adjustment of antibiotic treatment strategies}

This survey investigated the details of the choice of alternative antibiotics when the primary antibiotic was not effective (table 4). As shown in table 4, when NEC was confirmed, carbapenems were most commonly selected (43.0\%, including single or combined use), followed by antianaerobic agents, including metronidazole, ornidazole and clindamycin. Only $1.4 \%$ used antifungal agents as the first choice. When a patient's condition worsens or the treatment effect is unsatisfactory, most physicians switch to carbapenems.

\section{DISCUSSION}

\section{Selection of appropriate antibiotic regimens}

Broad-spectrum antibiotic therapy is an important treatment for all infants with NEC. However, the current status of antibiotic treatment of NEC in China remains unclear because there have been no previous reports. This study is the first to investigate the antibiotic use strategies employed by Chinese physicians for the treatment of NEC. The questionnaire survey had a wide geographical distribution and high coverage. The respondents were 284 pediatricians working in 182 tertiary hospitals in 29 provinces of China, most of them had worked in clinical practice for over 10 years, and more than half of the subjects had worked for over 20 years. The respondents have rich clinical experience, and their opinions are generally representative.

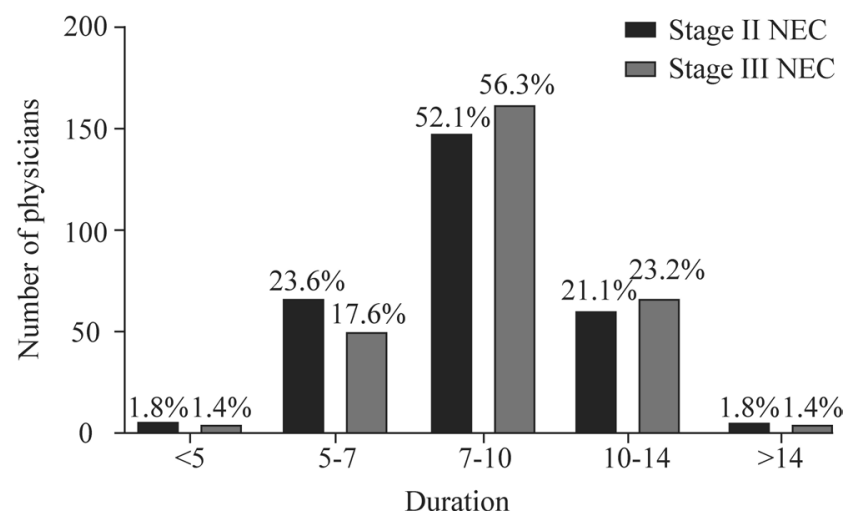

Figure 3 Duration of antibiotic therapy for stage II and stage III necrotizing enterocolitis (NEC) (days).
Based on the results of our questionnaire survey, $41.5 \%$ of the physicians chose beta-lactamase inhibitors, $28.9 \%$ chose carbapenems, and $20.8 \%$ chose cephalosporins to treat NEC among the single-antibiotic regimens. When combining antibiotics to treat NEC, beta-lactamase inhibitors and antianaerobic agents were the most frequently selected combination, followed by carbapenems and glycopeptides and cephalosporins and antianaerobic agents. There was no significant difference in the choice of NEC antibiotics by physicians with different durations of experience $(p>0.05)$. In Europe and the USA, triple antibiotic regimens are often used to treat NEC, such as ampicillin, gentamicin, and metronidazole,,${ }^{9} 12$ whereas in China pediatricians mainly select combinations of two antibiotics, primarily those effective against Gram-negative bacilli and anaerobic bacteria, which means that the treatment of Gram-positive cocci may be inadequate. In addition, cephalosporins are still effective against anaerobic bacteria, and it is not entirely reasonable to combine cephalosporins with metronidazole. In this survey, although there were as many as 20 reported combinations of antibiotics, the appropriateness of these combinations deserves consideration. This suggests that further study on the optimal combination

\begin{tabular}{|c|c|c|}
\hline Group & Antibiotic regimen & $\begin{array}{l}\text { Frequency, n } \\
\text { (\%) }\end{array}$ \\
\hline \multirow[t]{2}{*}{1} & Carbapenems as a first choice & $122(43.0)$ \\
\hline & $\begin{array}{l}\text { Step up to carbapenems after } \\
\text { deterioration }\end{array}$ & $135(83.3)$ \\
\hline \multirow[t]{2}{*}{2} & Glycopeptides as a first choice & $54(19.0)$ \\
\hline & $\begin{array}{l}\text { Step up to glycopeptides after } \\
\text { deterioration }\end{array}$ & $48(20.9)$ \\
\hline \multirow[t]{2}{*}{3} & $\begin{array}{l}\text { AAAs (metronidazole/ } \\
\text { ornidazole/clindamycin) as a } \\
\text { first choice }\end{array}$ & $100(35.2)$ \\
\hline & Add AAAs after deterioration & $13(7.1)$ \\
\hline \multirow[t]{2}{*}{4} & $\begin{array}{l}\text { Antifungal therapy included in } \\
\text { the initial combination }\end{array}$ & $4(1.4)$ \\
\hline & $\begin{array}{l}\text { Add antifungal therapy after } \\
\text { deterioration }\end{array}$ & $5(1.8)$ \\
\hline
\end{tabular}

AAAs, antianaerobic agents; NEC, necrotizing enterocolitis. 
of antibiotics is needed based on expert consensus or updated recommendations.

The American Surgical Infection Society and the Infectious Diseases Society of America issued guidelines in 2010 which suggest that broad-spectrum antibiotics, including ampicillin, metronidazole, and gentamicin; ampicillin, metronidazole, and cefotaxime; and meropenem, may be effective for the treatment of NEC in neonates. ${ }^{8}$ In addition, when methicillin-resistant Staphylococcus aureus or ampicillin-resistant infection is suspected, the guidelines recommend the use of vancomycin instead of ampicillin. ${ }^{8}$ However, the use of gentamicin is prohibited by law due to the adverse effects of ototoxicity and nephrotoxicity in children under 6 years old. In addition, according to the investigations into antibiotic resistance in recent years, the levels of resistance to penicillin and ampicillin are extremely high, ${ }^{15} 16$ and these antibiotics are not used for the treatment of NEC in China. Foreign retrospective studies have reported that antianaerobic therapy has no added benefit with regard to mortality due to NEC, nor can it prevent progression to surgical NEC. ${ }^{17}$ Researchers also retrospectively evaluated the effect of broad-spectrum antibiotics combined with metronidazole in the prevention of the progression of NEC from stage II to III, eventually concluding that the addition of metronidazole did not prevent the progression of NEC. ${ }^{18}$ Therefore, whether metronidazole, ornidazole, clindamycin, and other antianaerobic agents should be routinely included in the treatment of NEC is still controversial, and a large-scale, multicenter, prospective study is needed.

The etiology of NEC remains unclear, and recent studies have reported that it is related to genetic susceptibility, intestinal immaturity, hemodynamic instability, intestinal microbial imbalance and inflammation. ${ }^{19}$ There are distinct microorganisms responsible for NEC outbreaks; thus, it is clear that NEC is not caused by one species alone. ${ }^{20} \mathrm{~A}$ wide range of pathogens are associated with NEC; among anaerobic bacteria, some toxigenic Clostridium strains, such as Clostridium perfringens, C. difficile and C. butyricum, are implicated as causative pathogens in NEC. ${ }^{21-23}$ Although the specific pathogens responsible for NEC may affect the selection of antibiotic regimens, there is no established standard to suggest a benefit from a wider range of antibiotics. Additionally, the intestinal microbiota in newborns are easily affected by the environment. Given the differences among NICUs and antibiotic strategies, the environmental flora are likely to differ, ${ }^{24}$ which may lead to different pathogenic bacteria causing NEC.

\section{Duration of antibiotics}

Antibiotic therapy is variable. The use of antibiotics may not be effective if the duration of treatment is insufficient, while the prolonged use of antibiotics may result in an imbalance in the intestinal microecology and adverse consequences. ${ }^{25-27}$ However, there is no uniform global standard for the appropriate duration of antibiotic therapy. The Infectious Diseases Society of America suggests a duration of 4-7 days with adequate source control for complicated intra-abdominal infections, ${ }^{8}$ while 10 days is the duration recommended by the WHO ${ }^{28}$ Some studies have indicated that limiting antibiotic exposure to $7-10$ days might be optimal. ${ }^{29}$ Among the participants in this study, most chose 7-10 days for stage II NEC and 7-14 days for stage III NEC. There was no significant difference in the selected duration of therapy among physicians with different durations of experience, suggesting that Chinese doctors have a consistent understanding of the appropriate duration of antibiotic treatment.

\section{Adjustment of the antibiotic therapy strategies}

Owing to the delay in receiving blood culture results and to low positive rates, ${ }^{30}$ the treatment of NEC in China is mostly empirical. This survey showed that when NEC was confirmed, carbapenems were the initial antibiotic therapy selected, followed by the addition of antianaerobic agents (including metronidazole, ornidazole or clindamycin) and glycopeptides. When the therapeutic effect was unsatisfactory, the proportion of doctors who chose to escalate therapy with carbapenems was $83.3 \%$, and the proportion who chose glycopeptides was $20.9 \%$. The proportion who selected antianaerobic agents as the initial antibiotic regimen was $35.2 \%$; consequently, only $7.1 \%$ chose a combination regimen when NEC was exacerbated. When the treatment was ineffective, many physicians preferred to upgrade to carbapenems due to their stronger antibacterial effect on Gram-negative bacteria and due to their enhanced ability to act against anaerobic bacteria. In medical therapy for NEC in other countries, third-generation cephalosporins, carbapenems, and vancomycin are typically selected after empirical treatment with metronidazole. ${ }^{12}$

According to the results of this questionnaire survey, the antibiotics selected for the treatment of NEC in China are most commonly effective against Gram-negative bacilli. When the therapeutic effect is poor, carbapenems are primarily selected, and some physicians choose to combine carbapenems with metronidazole, ornidazole, and other antianaerobic agents. At present, in China, due to the laws and various medication-prescribing preferences, there is substantial variation in the empirical antibiotics selected for the treatment of NEC; in particular, the treatment of Gram-positive bacteria may be insufficient.

In conclusion, there is no unified antibiotic treatment program for NEC, and it is necessary to carry out multicenter research to serve as the basis for the formulation of relevant guidelines for the antibiotic treatment of NEC in the future.

Acknowledgements We thank all the physicians who participated in the questionnaire in this study.

Contributors LB contributed to conceptualization. YH involved in data curation. ZZ contributed to writing —original draft preparation. ZW and LL contributed 
to writing — review and editing. YS was in charge of supervision. All authors contributed to the article and approved the submitted version.

Funding This work was supported by the Chongqing Municipal Administration of Human Resources and Social Security (Cx2017107), the National Natural Science Foundation of China (82001602), the Natural Science Foundation Project of Chongqing Science and Technology Commission (grants cstc2019jcyj-msxmX0169 and cstc2018jscx-msyb-X0027) and the Science and Health Project of Chongqing Health Commission (2020FYYX217).

Competing interests None declared.

Patient consent for publication Not required

Ethics approval This study received ethics approval from the Institutional Review Board of the Children's Hospital of Chongqing Medical University (approval number 2020-22).

Provenance and peer review Not commissioned; externally peer reviewed.

Data availability statement All data relevant to the study are included in the article or uploaded as supplementary information. Data are available on reasonable request.

Open access This is an open access article distributed in accordance with the Creative Commons Attribution Non Commercial (CC BY-NC 4.0) license, which permits others to distribute, remix, adapt, build upon this work non-commercially, and license their derivative works on different terms, provided the original work is properly cited, appropriate credit is given, any changes made indicated, and the use is non-commercial. See: http://creativecommons.org/licenses/by-nc/4.0/.

\section{REFERENCES}

1 Brook I. Microbiology and management of neonatal necrotizing enterocolitis. Am J Perinatol 2008;25:111-8.

2 Stoll BJ, Hansen NI, Bell EF, et al. Trends in care practices, morbidity, and mortality of extremely preterm neonates, 1993-2012. JAMA 2015;314:1039-51.

3 Flahive C, Schlegel A, Mezoff EA. Necrotizing enterocolitis: updates on morbidity and mortality outcomes. $J$ Pediatr 2020;220:7-9.

4 Murthy K, Yanowitz TD, DiGeronimo R, et al. Short-term outcomes for preterm infants with surgical necrotizing enterocolitis. J Perinatol 2014;34:736-40.

5 Han SM, Knell J, Henry O, et al. Long-term outcomes of severe surgical necrotizing enterocolitis. J Pediatr Surg 2020;55:848-51.

6 Hansen ML, Jensen IV, Gregersen R, et al. Behavioural and neurodevelopmental impairment at school age following necrotising enterocolitis in the newborn period. PLOS One 2019;14:e0215220.

7 Neu J, Walker WA. Necrotizing enterocolitis. N Engl J Med 2011;364:255-64

8 Solomkin JS, Mazuski JE, Bradley JS, et al. Diagnosis and management of complicated intra-abdominal infection in adults and children: guidelines by the surgical infection Society and the infectious diseases Society of America. Clin Infect Dis 2010;50:133-64.

9 Shah D, Sinn JKH. Antibiotic regimens for the empirical treatment of newborn infants with necrotising enterocolitis. Cochrane Database Syst Rev 2012;8:CD007448.

10 Zani A, Eaton S, Puri P, et al. International survey on the management of necrotizing enterocolitis. Eur J Pediatr Surg 2015;25:27-33.
11 Blackwood BP, Hunter CJ, Grabowski J. Variability in antibiotic regimens for surgical necrotizing enterocolitis highlights the need for new guidelines. Surg Infect 2017;18:215-20.

12 Murphy C, Nair J, Wrotniak B, et al. Antibiotic treatments and patient outcomes in necrotizing enterocolitis. Am J Perinatol 2020;37:1250-7

13 Valpacos M, Arni D, Keir A, et al. Diagnosis and management of necrotizing enterocolitis: an international survey of neonatologists and pediatric surgeons. Neonatology 2018;113:170-6.

14 Bell MJ, Ternberg JL, Feigin RD, et al. Neonatal necrotizing enterocolitis. Therapeutic decisions based upon clinical staging. Ann Surg 1978;187:1-7.

15 Xie J, Peters BM, Li B, et al. Clinical features and antimicrobial resistance profiles of important Enterobacteriaceae pathogens in Guangzhou representative of Southern China, 2001-2015. Microb Pathog 2017;107:206-11.

16 Xiao YH, Wang J, Li Y, et al. Bacterial resistance surveillance in China: a report from Mohnarin 2004-2005. Eur J Clin Microbiol Infect Dis 2008;27:697-708.

17 Autmizguine J, Hornik CP, Benjamin DK, et al. Anaerobic antimicrobial therapy after necrotizing enterocolitis in VLBW infants. Pediatrics 2015;135:e117-25.

18 Luo LJ, Li X, Yang KD, et al. Broad-Spectrum antibiotic plus metronidazole may not prevent the deterioration of necrotizing enterocolitis from stage II to III in full-term and near-term infants: a propensity score-matched cohort study. Medicine 2015;94:e1862.

$19 \mathrm{Neu}$ J. Necrotizing enterocolitis: the mystery goes on. Neonatology 2014;106:289-95.

20 Zubarioglu U, Uslu S, Bulbul A. New frontiers of necrotizing enterocolitis: from pathophysiology to treatment. Health 2017:09:106-23.

21 Grishin A, Papillon S, Bell B, et al. The role of the intestinal microbiota in the pathogenesis of necrotizing enterocolitis. Semin Pediatr Surg 2013;22:69-75.

22 Schönherr-Hellec S, Aires J. Clostridia and necrotizing enterocolitis in preterm neonates. Anaerobe 2019;58:6-12.

23 Cassir N, Benamar S, Khalil JB, et al. Clostridium butyricum strains and dysbiosis linked to necrotizing enterocolitis in preterm neonates. Clin Infect Dis 2015;61:1107-15.

24 Hartz LE, Bradshaw W, Brandon DH. Potential NICU environmental influences on the neonate's microbiome: a systematic review. Adv Neonatal Care 2015;15:324-35.

25 Schulfer A, Blaser MJ. Risks of antibiotic exposures early in life on the developing microbiome. PLoS Pathog 2015;11:e1004903.

26 Cotten CM. Adverse consequences of neonatal antibiotic exposure. Curr Opin Pediatr 2016;28:141-9.

27 Fjalstad JW, Esaiassen E, Juvet LK, et al. Antibiotic therapy in neonates and impact on gut microbiota and antibiotic resistance development: a systematic review.. J Antimicrob Chemother 2018;73:569-80.

28 WHO Recommendations on Newborn Health. The who guide-lines review Committee/The World Health organization website (published 2017), 2018. Available: https://www.who.int/publications/i/item/ WHO-MCA-17.07 [Accessed 2 May 2017].

29 Cotten CM, Taylor S, Stoll B, et al. Prolonged duration of initial empirical antibiotic treatment is associated with increased rates of necrotizing enterocolitis and death for extremely low birth weight infants. Pediatrics 2009;123:58-66.

30 Connell TG, Rele M, Cowley D, et al. How reliable is a negative blood culture result? Volume of blood submitted for culture in routine practice in a children's Hospital. Pediatrics 2007:119:891-6. 